\title{
CONSTRUÇÃO E VALIDAÇÃO DE INSTRUMENTO PARA CONSULTA DE ENFERMAGEM NAS DOENÇAS CRÔNICAS NÃO-TRANSMISSÍVEIS (DCNT) CARDIOMETABÓLICAS NA ATENÇÃO PRIMÁRIA
}

\section{Maria Clara Moreira Matias*, Thaís Moreira São-João.}

\section{Resumo}

Dada a relevância do incremento do diabetes mellitus e da hipertensão em meio à população brasileira e mundial, é premente potencializar e efetivar a atuação do enfermeiro junto a esses pacientes. Trata-se de estudo metodológico, em desenvolvimento, que visa à criação e validação de instrumento para realização de Consulta de Enfermagem na Atenção Primária à Saúde; desenvolvido em Unidade Básica de Saúde (UBS) no interior do Estado de São Paulo e composto por duas etapas. A Etapa 1 corresponde à Construção do Instrumento e Validação de Conteúdo por especialistas e a Etapa 2 ao pré-teste e avaliação das propriedades de medida do questionário. Até o presente momento, foi executada a Etapa 1. Posteriormente, o questionário será aplicado a 40 pacientes com hipertensão arterial e/ou com diabetes mellitus tipo 2, usuários da UBS, como preconizado por diretrizes internacionais para condução de estudos metodológicos. Espera-se que esse estudo traga contribuições à Enfermagem à medida que disponibilizará um instrumento abrangente e prático

\section{Palavras-chave:}

enfermagem, questionários, doenças crônicas.

\section{Introdução}

Dada a magnitude do acometimento e crescimento da hipertensão arterial (HA) e do diabetes melittus tipo 2 (DM2) no Brasil e no mundo ${ }^{1}$, se faz necessário potencializar e efetivar a atuação do enfermeiro junto a esses pacientes.

Trata-se de estudo metodológico que tem como objetivo a criação e validação de um instrumento para Consulta de Enfermagem de pacientes com HA e/ou DM2 em seguimento na Atenção Primária composto por duas etapas em Unidade Básica de Saúde (UBS) no interior do Estado de São Paulo. A Etapa 1 corresponde à Construção do Instrumento e Validação de Conteúdo por especialistas e a Etapa 2, ao pré-teste e avaliação das propriedades de medida.

\section{Resultados e Discussão}

Até o presente momento, foi executada a Etapa 1, que gerou um breve instrumento de duas laudas que reúne dados quanto a:

(1) Caracterização sociodemográfica e clínica, hábitos e estilo de vida e exame físico;

(2) Diagnósticos de Enfermagem (DE), tendo sido levantados pela pesquisadora 33 DE prontuários de pacientes em seguimento na UBS entre janeiro de de 2017 e fevereiro de 2018; baseados na Classificação Internacional para a Prática de Enfermagem (CIPE); e (3) Resultados esperados e ações de enfermagem.

A Etapa 2, a ser iniciada em julho de 2018, contará com o pré-teste, que corresponde à aplicação do questionário a 40 pacientes com HA e/ou DM2, usuários da UBS, como preconizado pelas diretrizes internacionais.

\section{Conclusões}

Após levantamento dos principais Diagnósticos de Enfermagem vinculados aos pacientes com HA e/ou DM2 em acompanhamento na Atenção Primária, foi reafirmada a necessidade de um instrumento validado para estruturação da Consulta de Enfermagem a tais pacientes pela dificuldade da utilização da Classificação Internacional para a Prática de Enfermagem (CIPE) ${ }^{2}$ pelos enfermeiros, 0 que facilitará um efetivo acompanhamento e reconhecimento do estado de saúde do paciente pela equipe de saúde, assim como reconhecimento do trabalho do enfermeiro pelo registro do raciocínio clínico e intervenções efetuados.

\section{Agradecimentos}

PIBIC/CNPq - Bolsa IC - Quota 2017-18.

\footnotetext{
1 American Heart Association. Heart Disease and Stroke Statistics-2012 Update: A Report From the American Heart Association. Circulation 2012; 125: e2-e220. DOI: 10.1161/CIR.0b013e31823ac046
}

${ }^{2}$ Garcia TR (Org.). Classificação Internacional para a Prática de Enfermagem (CIPE®): versão 2015. Porto Alegre: Artmed, 2016. 270p. 